\title{
Resilience engineering in practice: Reflecting on a pediatric hospital's preparation for unknown coronavirus outbreak
}

\author{
Shanqing Yin, Chia Yin Chong, Kee Chong Ng, Khai Pin Lee \\ KK Women's \& Children's Hospital, Singapore
}

Received: October 20, 2020

Accepted: November 12, 2020

Online Published: November 27, 2020

DOI: $10.5430 /$ jha.v9n6p1

URL: https://doi.org/10.5430/jha.v9n6p1

\begin{abstract}
At the start of 2020, hospitals around the world were trying to adapt during the COVID-19 pandemic. From the resilience engineering perspective, this outbreak would be a significant test as healthcare institutions try to tolerate and manage this major disruption. This paper shares insights on what a stand-alone paediatric hospital in Singapore had done to stay ahead since the beginning of the outbreak. Observations were conducted from 25-Jan-20 to 25-Mar-20 to capture evidence of resilient behavior, notably in the form of improvisations. Findings revealed adaptations made across various organization levels: at the macrosystem to create capacity to isolate safely, at the mesosystem to facilitate teamwork, and at the microsystem to manage compromises at the frontlines. Juxtaposing this episode with other examples of organizational resilience, this paper maps out common resilience engineering themes in the hospital's response to COVID-19, but also questions what defines an organization's success in being resilient.
\end{abstract}

Key Words: COVID-19, Resilience engineering, Hospital management, Pandemic preparation

\section{INTRODUCTION}

Resilience and resilience engineering investigate how individuals, teams and organizations monitor, adapt to and act on failures in high-risk situations. ${ }^{[1]}$ Resilience engineering resonates with many complex, high-risk industries such as aviation, transportation, manufacturing \& production, and especially in healthcare. ${ }^{[2]}$ Behaviors in complex systems are hard to predict, and perturbations in one locale can cause a ripple effect to another area unrelated in time or place. ${ }^{[3]}$ Healthcare in particular features emergency departments and fast changing acute care facilities that require the ability to adapt to various complexity and unpredictability. ${ }^{[4,5]}$

There are many perspectives to understand a resilient system.
The Resilience Analysis Grid ${ }^{[6]}$ noted four abilities of how a system responds, monitors, learns, and anticipates changing situations. A system is also deemed resilient if it can adjust its functioning before, during, or following events (e.g. changes, disturbances, or opportunities) and thereby sustain required operations under both expected and unexpected conditions. ${ }^{[7]}$ Other descriptions of resilient performance include being robust, not brittle, able to rebound back to equilibrium from surprises and trauma, and adaptive for a sustained period in the face of uncertain, dynamic conditions. ${ }^{[8]}$ Yet other studies highlighted the ability to cope with complexity while balancing productivity with safety, ${ }^{[9]}$ manage risks proactively, ${ }^{[10]}$ and be resistant to operational loss. ${ }^{[11]}$ The list goes on.

*Correspondence: Shanqing Yin; Email: shanqing.yin@gmail.com; Address: KK Women’s \& Children’s Hospital, Singapore. 
A system's resilience is most visible when it responds to internal and external perturbations. Disruptions may be internal such as equipment malfunction or manpower on sick leave, as well as external like storms or major political events Disruptions may be routine (network server downtime), regular (influenza season), or rare (terrorist attack). Disruptions are most stressful when they are sudden, unplanned and unexpected, progressing quickly, and possibly costly. Ideal systems are expected to absorb and accommodate these stresses (i.e. being prepared to adapt to handle surprises), or at least go into graceful and controlled degradation so as to minimize negative impact. ${ }^{[8]}$

Sudden major disruptions are the most challenging, typically involving multiple confounding variables, intense pressure, and costly consequences. ${ }^{[12]}$ Unlike noisy but normal operations, sudden, rare, destructive events like industrial fires, hurricanes, and military conflicts can significantly tip any system off-balance. These situations generate stress loads that systems may not be familiar with or structurally-designed to endure. The system may fracture as a result, unable to adapt or sustain operations, or may not recover back to its former glory. Such traumatic surges are true tests of resilience and dynamic adaptation, as seen in Sunrise Hospital during the 2017 Las Vegas Mass Shooting, ${ }^{[13,14]}$ or the medical response during 2015 Formosa Fun Coast Dust Explosion in New Taipei City. ${ }^{[15]}$

More recently, the world-wide COVID-19 pandemic was a major evolving disruption which confronted many healthcare systems around the world. At the beginning, this outbreak offered limited information for hospital management to work on, with new struggles and changing situations further challenging healthcare institutions to adapt in order to continue delivering care safely and effectively.

This paper reviews some of the common themes behind resilience engineering, and juxtaposes them with a pediatric hospital's reaction to a major pandemic of unknown etiology. It further provides some insights into how resilience engineering may look like in practice.

\section{BACKGROUND}

\subsection{Resilience is being proactive and preemptive}

A resilient organization, team, or system is often described as being able to "stay ahead of the curve", in that it seems able to anticipate and withstand varying and increasing load. A less desirable approach is to react after changes have occurredalthough an equally acceptable outcome is possible, it entails more effort and risk. Resilience engineering takes into account preparatory efforts to mitigate impending or unexpected disruptions, possibly preventing future problems. ${ }^{[16]}$
A resilient system thus should anticipate, learn, and adapt, ever-ready for emergencies during normal operations and when under stress. ${ }^{[12]}$

Proactive behaviors facilitate success in responding to sudden demands and disruptions. Prior to the arrival of the first Las Vegas Casualty shooting to Sunrise Hospital, the emergency department rallied all available manpower and surplus supplies, and freed up all operating rooms. ${ }^{[13]}$ Simulation exercises during "peace time" prepared teams to triage and treat victims timely during actual mass casualty events, ${ }^{[17]}$ such as during the Boston Marathon bombing. ${ }^{[18,19]}$ Proactive behaviors are deliberate, and requires additional effort and resources beyond normal operating requirements.

\subsection{Resilience requires resources}

Resilience is facilitated by keeping additional resources readily available. Tension and trade-offs usually arise due to limited resources, as demand begins to stretch existing supply. Resources are hardest to come by when needed the most, and resilient organizations standby reserves, generate new capabilities (e.g.: repurposing spaces), and tap on alternative supplies (e.g.: mobilizing office administrators to help). Resilience involves effective adaptation, and effective adaptation requires access to space, equipment, manpower, and expertise.

Thus, analyzing an organization, team, or individual's ability to be resilient can partly be inferred through the availability and accessibility of such resources. Undesirable outcomes may not always be due to missteps or failures, but also inappropriate or insufficient system adjustments. ${ }^{[6,20]}$

\subsection{Resilience is timely, decentralized decision-making} Success involves more than just prescriptive orders from a central command. ${ }^{[21]}$ A rigid, bureaucratic administration during a dynamic crisis can complicate communication and hinder situation awareness. These issues are frequently cited in Hurricane Katrina case studies. ${ }^{[23,24]}$ Simply executing a disaster preparedness plan is insufficient. Instead, generating ad-hoc resolutions early in the crisis can prevent deterioration. Decisions on the ground have the benefit of minute and up-to-date information, and are more sensitive to the compromises and tradeoffs that significantly affect frontline operations.

Decentralized control promotes rapid decision-making during crisis. However, it requires autonomy and creativity, especially when improvising. ${ }^{[23,24]}$ In the immediate aftermath of the September 11 World Trade Center attacks, approximately 500,000 people left Manhattan via a variety of boats: tour boat, military vessel, passenger ferry, or even private craft. ${ }^{[25]}$ This unorthodox process was devised amid the city- 
wide evacuation. Expert decision-making were disseminated by empowering Coast Guard inspectors exercise discretion.

\subsection{Resilience involves managing trade-offs}

With finite resources, crisis response operates on a satisficer model, which naturally involves trade-offs. Moments of resilience often revolve around balancing conflicts or even taking chances. This has been captured in resilience engineering literature in the form of the efficiency-thoroughness trade-off principle (or ETTO principle) ${ }^{[26-28]}$ sacrifice judgments, ${ }^{[29]}$ or simply some kind of compromise between two or more conflicting intentions. ${ }^{[30,31]}$ Common conflicting goals include safety, productivity, and economic gains. ${ }^{[12]}$ Even day-to-day clinical work involves managing dilemmas and adaptations so as to meet changing operational goals. ${ }^{[32]}$

\subsection{Resilience is cultural}

Resilience Engineering has been advocated as a core component in an organization's safety culture. ${ }^{[16]}$ Purportedly, resilience can be intentionally designed into the system so as to boost its capability to deal with stresses. Yet much of what promotes resilience alludes back to an organization's established culture, mindset, and beliefs. These include concepts such as organizational commitment and involvement, employee empowerment, rewards system, safety values, making sacrifice judgments and willingness to temporarily relax operational goals. ${ }^{[33-35]}$

True to its cultural characteristic, a distinct feature of resilience is its emergent property. ${ }^{[36,37]}$ While some academics have advocated measurement tools to capture a system's resilience potential, true reflection of resilience appears in the form of reactions to an impact. ${ }^{[38]}$ Resilience goes beyond consolidating a combination of "resilient" initiatives,

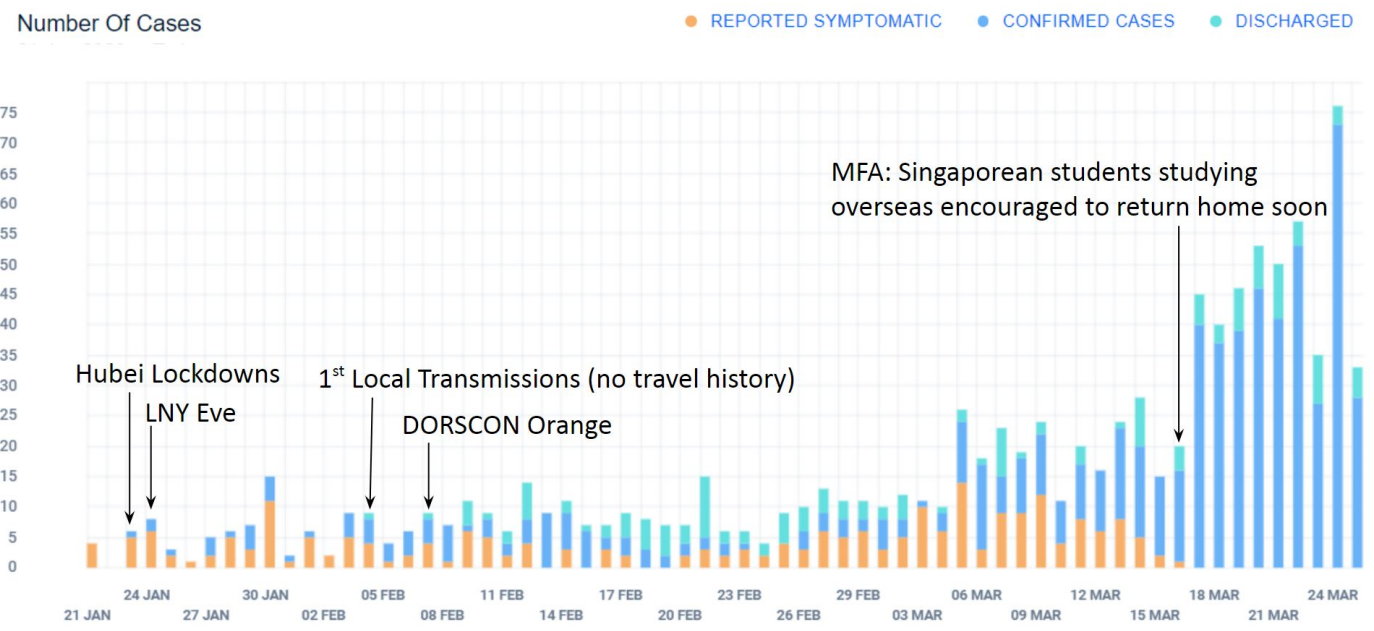

Figure 1. The number of cases related to COVID-19, categorized as "Reported Symptomatic", "Confirmed", and "Discharged", from 21-Jan to 25-Mar. Source: co.vid19.sg

Published by Sciedu Press 
Local transmissions began occurring in February, and the Singapore government activated Disease Outbreak Response System Condition (DORSCON) Orange on 7 Feb 2020. ${ }^{[40]}$ Orange is the second highest DORSCON alert level, with Green being the lowest followed by Yellow, and Red being the highest. By March, the WHO declared COVID-19 a global pandemic. The case definitions set by Singapore's Ministry of Health (MOH) were frequently updated, and even then hospitals played it safe by admitting more suspect cases, so that these cases were already kept safe in isolation if the tests came back positive. Possible infected persons were no longer limited to individuals with travel history to a specific city or country. By 17 March, the Ministry of Foreign Affairs urged Singaporean students studying abroad to return home.

With this initial wave of cases, the nation launched a strategy of containment with enhanced surveillance. All suspect cases as well as individuals with a high chance of being infected were immediately isolated from the public. Public hospitals were instructed to admit and isolate any suspected cases that were identified through the emergency services. As a result, while the number of positive cases was low, healthcare systems were stretched due to the high surge in suspect cases. All admitted cases were tested, and even if the results were negative, patients would remain in isolation until consecutive COVID-19 polymerase chain reaction (PCR) tests returned negative by the fifth day of the illness.

\subsection{Main care center for paediatric COVID-19 cases}

While the purpose-built, 330-bed National Centre for Infectious Diseases (NCID) in Singapore was the primary location for all adult suspect and confirmed cases during any disease outbreaks, KK Women's \& Children's Hospital (KKH) served a similar role for pediatric cases. As a specialist hospital focusing on women's and children's health, the hospital has over 800 beds including 52 isolation beds. One isolation ward has 12 beds, the pediatric ICU has 4 isolation beds and the remaining 36 isolation beds are scattered across various wards.

Like most other hospitals, KKH did not have vast dedicated facilities on standby to handle massive disease outbreaks. The pressure was on the hospital and its medical teams to adapt and absorb the sudden and unpredictable surges. Process and protocols based on past outbreaks like the H1N1 in 2009 and SARS in 2003 have already been implemented. Emergency preparedness exercises and drills had been previously conducted with $\mathrm{MOH}$, which included drills to exercise disease outbreak scenarios. Armed with these protocols and practices to guide responses, $\mathrm{KKH}$ adapted and improvised as the situation unfolded.

4

\section{Methods}

The use of ethnographic methods has been commonly adopted to collect insights of resilience as it manifests during situations. ${ }^{[4,21,41,42]}$ Ethnographic observations has been effective at capturing more descriptive, qualitative data such as how and why processes work out right, ${ }^{[29,43]}$ what improvisations look like, and what really happens on the ground as work-as-done instead of work-as-imagined. ${ }^{[44]}$ Ethnographic observations could be employed efficiently under short notice. Looking out for trade-offs helped to understand the difficulties decision-makers faced and guided the attention towards possible resilient responses. ${ }^{[45]}$

The Resilience Analysis Grid ${ }^{[6]}$ was used as a frame of reference to guide observations. While the Resilience Analysis Grid offered a tool to assess the potential of a system to be resilient, the goal of this study was to document what resilient behaviors might look like "in flight" in the midst of responding to a crisis. The four abilities of learning, responding, monitoring, and anticipating were nonetheless useful to frame the observed activities and facilitate further exploration.

KKH's resident human factors specialist began observing and documenting from 25 Jan 2020, and continued on until 25 Mar 2020, after which it was deemed that pediatric cases were relatively lower than adult cases, and the hospital felt they had the situation under control. The human factors specialist (author YSQ) was formally trained and possessed a $\mathrm{Ph}$.D. in human factors. Whilst his role over the 9 years of being embedded in healthcare centered around quality improvement and improving patient safety, the specialist served as a trained eye during this study period.

Depending on the theme of the data to be captured, observations were routine (e.g. monitoring a shift in the emergency department or isolation ward), scheduled (e.g. shadowing doctors during isolation ward rounds), or opportunistic (e.g.: patient journey upon admission from emergency department to the wards, resuscitation during a Code). Where necessary, clarifications were sought during opportunities when clinicians were available or no longer busy with the case. Data-collection will primarily focus on possible adaptations and trade-offs across three main tiers of organization, teams, and individuals.

\section{Results}

Incidentally, the results of the observations reflected how resilience can be found across different functional levels of the system, in the form of "micro-meso-macro". [46] The same resilience concepts, themes, and behaviors can be observed at the organizational level (macrosystem), within 
teams (mesosystem), and within individual actions (microsystem). While the findings aim to distinguish resilient efforts within each functional level, influences from other tiers above and/or below are relevant and inevitable.

\subsection{Macrosystem: Creating capacity to isolate safely}

There was an urgent need to ensure suspect cases were swiftly and safely isolated, as the SARS outbreak in 2003 revealed how hospitals were super-spreaders and healthcare providers were infected. ${ }^{[4]}$ Every suspect case had to be treated as a possible COVID-19 infection until confidently proven otherwise. Confounded by scant diagnostic details and similarity with other respiratory infections, the volume of people requiring COVID-19 attention was substantial, even if they eventually turned out to be false alarms. Two key areas were immediately affected by this surge, the Children's Emergency helming the triage, and the isolation wards receiving and housing the patients possibly with COVID-19.

KKH's Children Emergency (CE) had a small annex at the side designed and designated for infectious cases. The CE Annex consisted of three consult rooms, a larger treatment room, a pantry at the rear, and toilets. All consult and treat- ment rooms featured negative pressure. All staff in the annex would don "full" infection prevention personal protection equipment (PPE) of isolation gown, latex gloves, N95 respirator, face shield, and surgical cap.

Soon after learning of the outbreak in January, CE responded by expanding capacity to anticipate the demands for care, while ensuring safe segregation between different patient groups (i.e. suspect versus non-suspect cases). The initial wave of Chinese tourists seeking medical attention during the Lunar New Year weekend stretched the capacity of the CE Annex. As CE monitored the situation over the subsequent week, plans were made with hospital management to convert the open spaces outside of CE, normally set aside for mass chemical decontamination, into triage stations and outpatient clinics (see Figure 2). The waiting area within the CE was also divided by a temporary wall, with the adjacent consult rooms segmented accordingly to zones of varying infection risks. These zones were allocated based on the facilities they could accommodate, such as access to mobile $\mathrm{x}$-ray machines (initially pneumonia was a clinical feature of COVID-19) and negative pressure rooms.

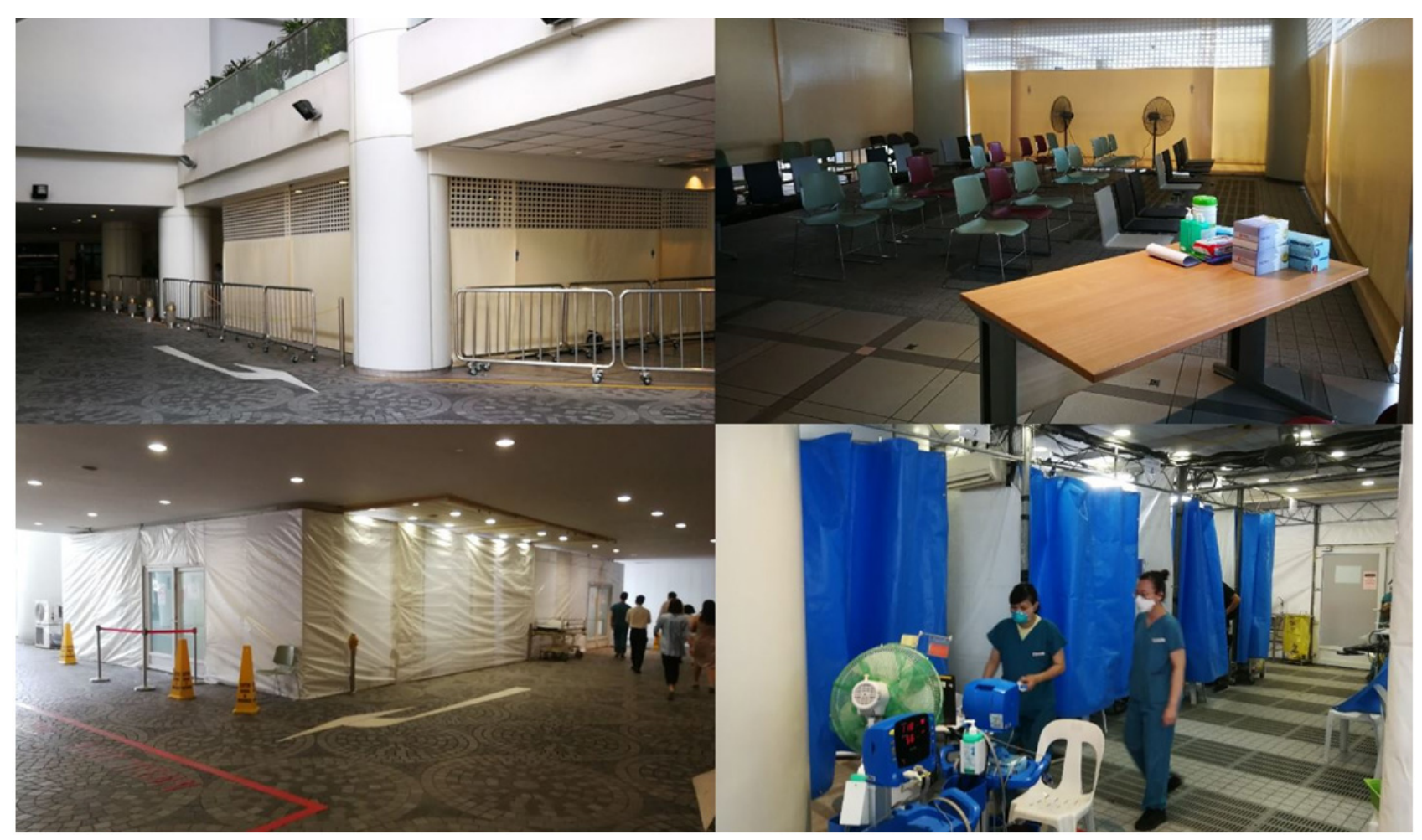

Figure 2. Spatial adaptations around the Children's Emergency

Top photos: Mass decontamination area was repurposed into additional triage and waiting spaces. Bottom photos: Large tents were erected near the drop-off drive way to serve as separate make-shift consult rooms away from existing infrastructure to minimize cross-infection. 


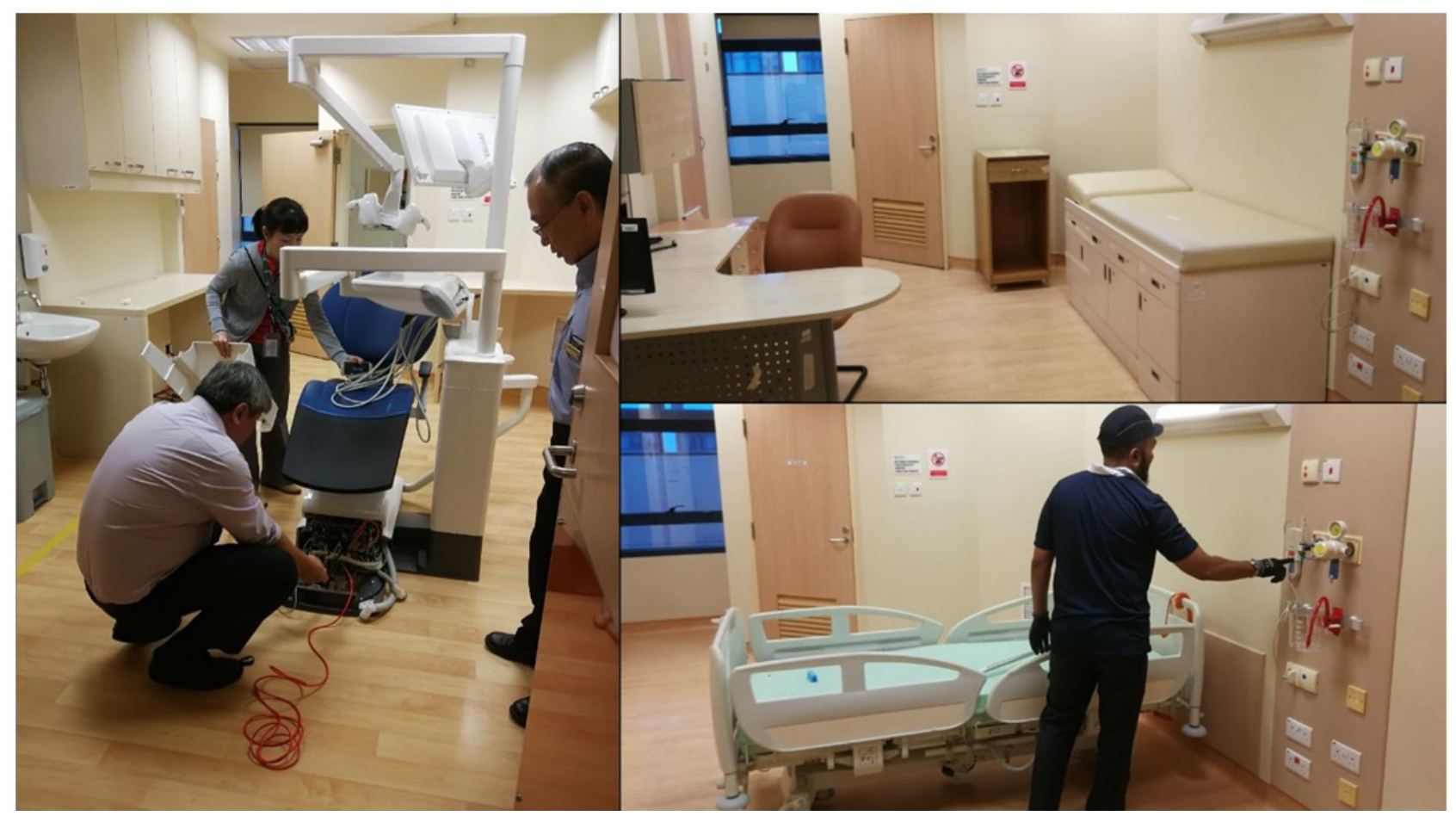

Figure 3. Converting outpatient spaces such as dental clinics and consult rooms into isolation units

Clockwise from left: Technicians disconnecting wiring and plumbing prior to shift; An outpatient consult room prior to modification;

Performing final tests in modified isolation room.

Anticipating the unpredictable surge, hospital management recognized that more isolation rooms were needed. In existence was a 12-bed isolation unit for pediatric patients. As a response, Premium wards with private rooms were decanted, and the rooms were refurbished to manage infectious diseases (e.g., donning \& doffing stations). Selected outpatient clinics were also repurposed into isolation wards, with some rooms requiring the removal of heavy permanent fixtures such as dental chairs and examination tables (see Figure 3 ). Five isolation units were eventually prepared to manage pediatric COVID-19 cases, with a total capacity of 40-50 isolation rooms. This allowed for dedicated nursing and medical teams to work on COVID-19 cases, consolidated and prioritized use of personal protective equipment, and ultimately reduced cross-infection risks between other hospital areas. Beyond monitoring the occupancy load, the concentrated services was also more efficient for management to monitor the deployment and protection of limited care resources.

Effective resilience required resources to contain the disruption, and existing manpower was reallocated to support the new isolation wards and other COVID-19 interventions. With DORSCON Orange, many elective surgical procedures were postponed, while precautionary measures like visitor restrictions and temperature screening were initiated. Other COVID-19 work included setting up a Hospital Command Center as well as contact tracing. Nurses from elective and less-essential services were redeployed to beef up staffing at clinical hot zones. Pediatric doctors were rostered into weekly 12-hour shifts to cover the COVID-19 wards. Parttime hires manned screening stations, supported by administrative staff from various back-office departments.

Perturbances to the system would ripple through and affect other components, and the increased number of doctors on overnight shifts resulted in a need for places that these doctors could rest. The hospital already had on-call rooms for various physicians rostered for night duty, but these were insufficient for the doctors supporting the COVID-19 wards. Creatively, some of the doctors decided to use the medical education seminar room as a makeshift accommodation. They eventually got in touch with relevant departments to help furnish the space. Figure 4 shows the medical education seminar room adapted into a shared quarters, with rollaway beds and storage space for doctors on shift that night. This was one of many incidents of decentralized decision-making that took place within and between teams.

\subsection{Mesosystem: Strategies to facilitate teamwork}

The novel coronavirus outbreak introduced an unknown threat, with case definitions constantly changing over a couple of days. What began in late January as "symptoms suggestive of pneumonia" which would rely on chest x-rays 
for diagnosis, eventually became a more generalized "acute respiratory infection" as community spread became more wide-spread. $\mathrm{MOH}$ circulars on suspect case definitions changed 8 times from 2-Jan to 9-Mar (2 more COVID-19 circulars were subsequently released in April, and one in May), with changes happening every other day from 21-Jan leading up to the Lunar New Year of 25-Jan (i.e. 3 changes in 5 days).

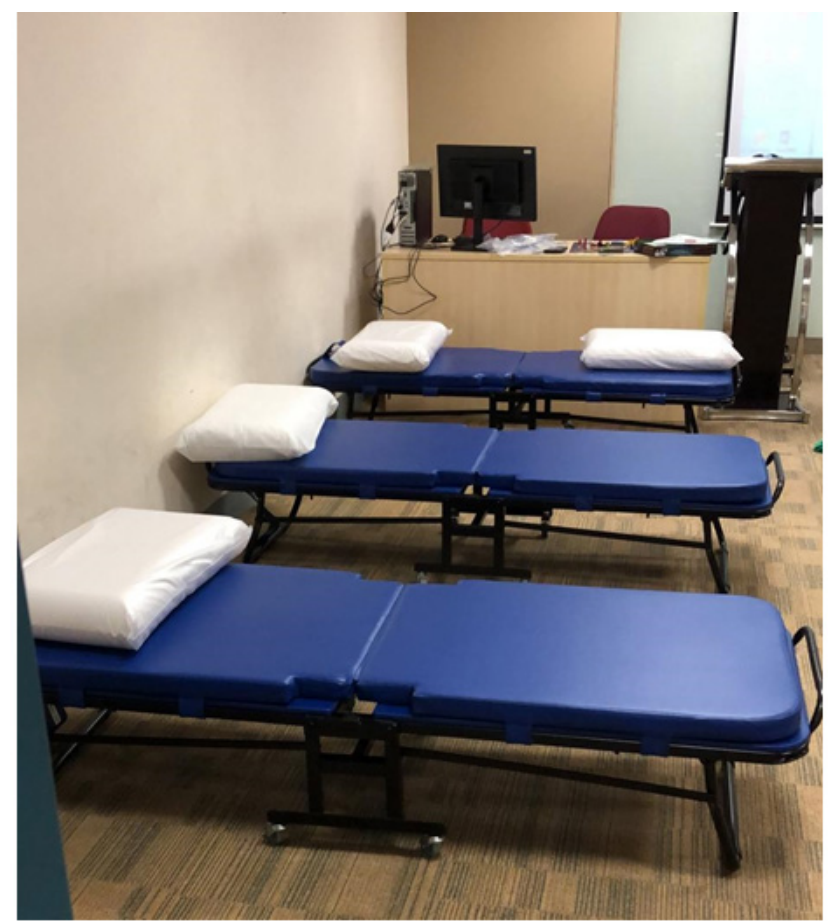

Figure 4. Rollaway beds were placed within a seminar room to serve as shared sleeping quarters as physicians took on 12-hour shifts

Although all doctors would receive up-to-date circulars from the Ministry, physicians in both emergency medicine and infectious disease domains remained alert to constantly learn new information about the virus. To support fellow doctors in their clinical triages of suspect COVID-19 cases, the hospital's Infectious Disease (ID) physicians responded by setting up a dedicated hotline using a mobile phone for all doctors to call and consult. This hotline was manned by one ID physician dedicated to COVID-19 clinical issues, and this duty was rotated among the various ID physicians. This service afforded easy access to expertise and facilitated many COVID-19 concerns to be addressed promptly, although the team continued to monitor its use. The initiative did consume one dedicated ID manpower, but it allowed the remaining ID teams to focus on and better anticipate other existing and emerging concerns.
As the world continued to understand the "nature of the beast", new information meant sudden surprises that required expedited resolutions. The emergency resuscitation protocol (Code Blue) had to be reviewed due to aerosol-producing procedures such as intubation and suctioning. Rescuers were now at risk of being infected when chest compressions and ventilation were performed during cardio-pulmonary resuscitation (CPR). The cumbersome process of donning full personal protection equipment (PPE) consumed precious time. Responding to the hurdles learnt during the unknown coronavirus care delivery, Cold Blue clinical stakeholders such as critical care intensivists, airway management anesthetists, and pediatricians discussed, simulated, and disseminated updated the emergency resuscitation protocol for suspected and confirmed COVID-19 patients. Modifications included having preliminary protection for Code Blue "firstresponders" while the main team suited up, keeping the medication "crash cart" outside the isolation room to reduce contamination, and establishing a communication chain between the inside resuscitation team and the outside support team. Multi-disciplinary simulation re-trainings with the updated protocol were conducted.

Specifically, team member identification and communication during an infectious disease Code Blue was challenging. Everyone looked the same under the PPE. The PPE muffled voices. Communication between team members within and outside the isolation room was physically hindered by two walls and an ante room. Colleagues from the CE department who participated in the Code Blue simulation shared their creative practice of using role sticky labels such as "team leader", "medication nurse" and "airway doctor" to identify each team member's specialty and functional role. Such decision-making moments among teams and team members, coupled with the sharing of ideas and resources, expedited problem resolutions.

\subsection{Microsystem: Handling frontline compromises}

Many individual decisions were made during the course of managing the outbreak. At the Children's Emergency Annex, clinicians tried to keep the 3 private consult rooms available for the clinically more severe cases, by isolating patients who appeared unlikely of COVID-19 in larger "shared" spaces like the treatment room or even the staff pantry. While each consult room could only accommodate one patient case, it afforded the mobile x-ray machine to be brought in and used if necessary. The treatment room and pantry, on the other hand, although large enough to cohort patients, would require moving patients if $\mathrm{x}$-rays were required, thus increasing risk of infection spread. The consult room also provided radiation protection for everyone else in the small annex space. 
This strategy was a trade-off between the dangers of moving infectious patients around versus cohorting patients. As a mitigating strategy, patients who were cohorted in the larger spaces were kept distanced as much as possible.

Reusable air-tight safety goggles along the likes of ski masks were issued to each doctor and nurse working with COVID19 cases. With the dwindling supply of disposable face shields dwindling, safety goggles became part of the standard PPE. This posed a new challenge for clinicians to carry and store them safely. Existing zip-lock bags were too small and larger ones had to be procured. Carrying the goggles in the pockets felt bulky, so others innovatively used clear plastic boxes to house their goggles at workstations (Figure 5). Walking to and fro the workstation to retrieve and store goggles became a trade-off.

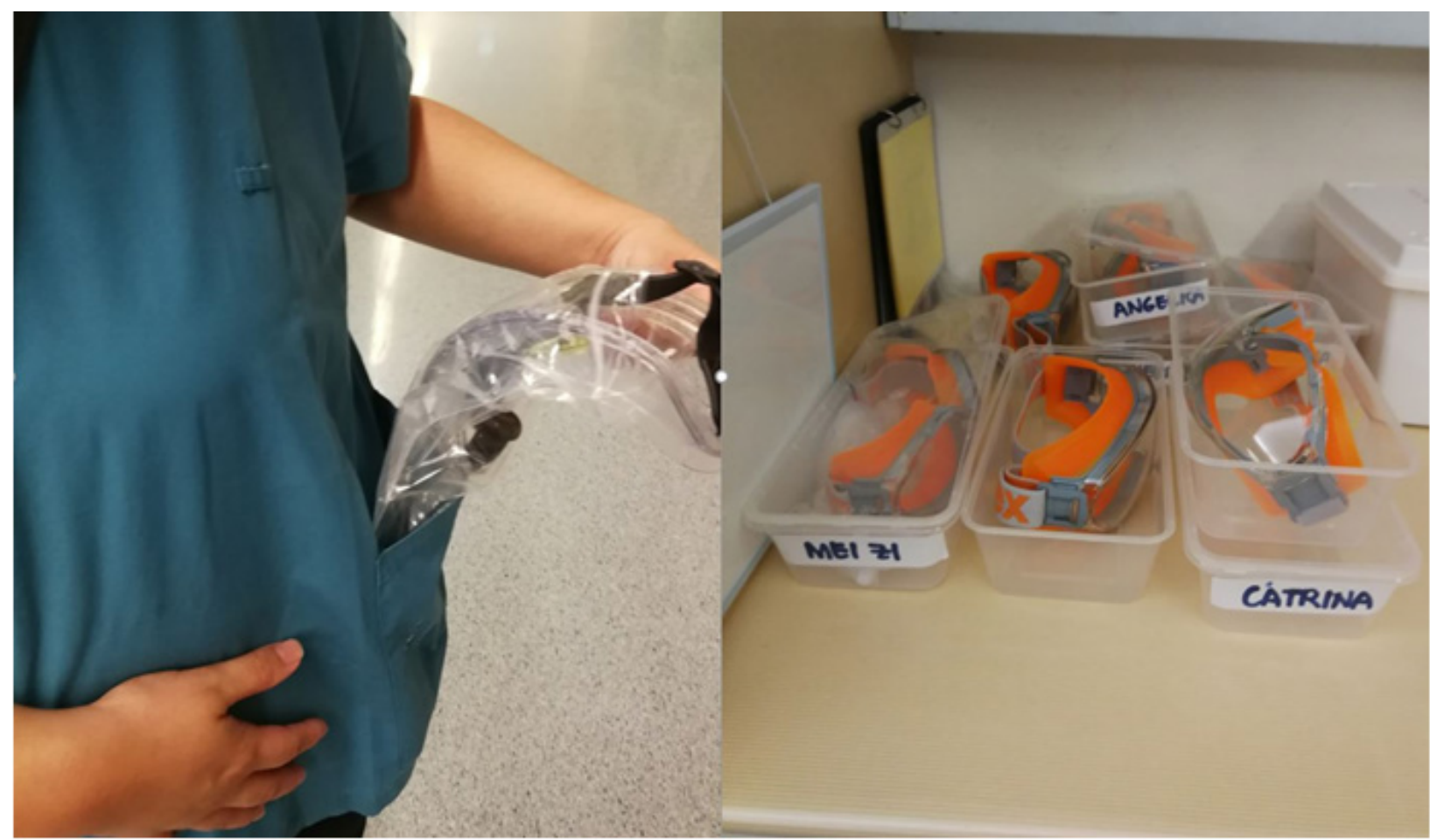

Figure 5. Clinicians had to find ways to safely store their protective goggles

Some kept in plastic sealable bags to be stored in their scrub suit pockets. Others stored their goggles in personalized plastic boxes on their workstations. Each option provided its own pros and cons.

During these early weeks of the pandemic, many clinicians chose not to go home, or opted to extend their presence at work. Singapore was hit by Severe Acute Respiratory Syndrome (SARS) in 2003, during which many healthcare professionals were infected by and even succumbed to the disease. It was then that clinicians adopted the idea of staying in hotels and away from family, to minimize unintended harm to their loved ones. Similar precautions were practiced during this pandemic, whereby colleagues might have booked a hotel room nearby, or distanced themselves when they got home until laundry and a good shower were performed. Other clinicians worked longer hours to provide additional support. While these choices meant less family and personal time, as well as increased effort to combat stress and fatigue, healthcare professionals recognized their calling and knew this would be their finest moment.

\section{Discussion}

This paper sheds light on resilience in action, by observing how a regular children's hospital in Singapore adapted to become the primary facility for all suspect and positive pediatric COVID-19 cases. Many established themes surrounding resilience were echoed in this case study, as well as some challenges to the current concepts of resilience and resilience engineering.

\subsection{Improvisations as manifestations of resilience}

Improvisations are cues to adaptive effectiveness, and should serve to qualitatively measure resilience. ${ }^{[4]}$ Improvisations can appear differently at various levels of resolution: organizational response, team performance, and individual improvisations. ${ }^{[48]}$ While very much interdependent, the pressures, decisions, and adaptations between an outbreak taskforce 
and a doctor at the frontline will be different. Analyzing these adaptations can reveal rich details such as conflicting goals (e.g. spatial separation of teams versus providing cross-coverage), mismatched resources, bottlenecks and challenges, and ultimately the system's adaptive capacity.

Improvisations also revealed how trade-offs tend to be emergent rather than planned. While organizations establish an overarching strategy (e.g.: keep suspect cases isolated), what actually takes place can differ due to situational factors (e.g.: cohort patients safely so as to keep private rooms available for more invasive procedures and higher-acuity cases). Many effective adaptations are ad-hoc and may not have been devised in the board room. Yet these individual decisions significantly influences the system's resilience capability, further suggesting that resilience may fundamentally be a cultural phenomenon (or climate) driven by the people within organizations. ${ }^{[16,49]}$ Administrators should not wait for another major outbreak or disaster to embark on a journey to cultivate a culture of organizational resilience.

Besides establishing a culture of resilience, organizations can promote success by facilitating effective improvisations during times of crisis. Improvisation is not simply allowing entities to act at will. Rather, it entails recognizing the tradeoffs, being aware of the risks, and where possible putting in place control measures. Such behaviors can be trained as well as honed through experience across the different functional levels of the system, from frontline managers all the way to executive leaders. After all, at the heart of effective improvisation are resilience fundamentals of being proactive, preemptive, and resourceful.

\subsection{Was KKH successful at being resilient?}

Is success in resilience engineering measured by a lack of failure? Failure may appear straightforward in a space shuttle disintegration, ${ }^{[10]}$ or hidden within the repercussions of trade- offs, such as mental health. ${ }^{[50]}$ A proactive and responsive system may still succumb if given sufficient magnitude of strain or attrition over time. How long should the evaluation window last? Is hindsight the only means to critique past foresight? Might the Fukushima nuclear plants had gotten away with "being resilient" had the tsunami waves been less than $5.7 \mathrm{~m} ?^{[51]}$ This paper adds to the existing challenges of studying resilience, in that while we think KKH has been successful at being resilient, we risk speaking too soon and jinxing ourselves.

Beyond the difficulty to define success, other limitations to this study include capturing only snapshots of the system in action. The one trained observer might have missed spotting other examples of resilience, as well as moments of failures. Repercussions from choices and trade-offs may not be immediately observed too.

Ultimately, the "principles of crisis management" differ from the "principles of disaster preparedness planning". ${ }^{[52]} \mathrm{Re}-$ silience engineering research advocates the constant facilitation for success as well as proactive preparation for disruptions. Resilience engineering in practice might refer less on past preparatory efforts, and more on emergent systemic behaviors which are revealed only when under pressure. Major crises such as COVID-19 are a true test of resilience on healthcare institutions, and one week in COVID-19 can be an excruciatingly long time.

\section{ACKNOWLEDGEMENTS}

We sincerely thank everyone at KKH who stayed adaptive and resilient in the face of uncertainty.

\section{CONFlicts of InTEREST Disclosure}

The authors declare they have no conflicts of interest.

\section{REFERENCES}

[1] Jeffcott SA, Ibrahim JE, Cameron PA. Resilience in healthcare and clinical handover. BMJ Quality \& Safety. 2009 Aug 1; 18(4): 25660. PMid: 19651927. https://doi.org/10.1136/qshc. 2008. 030163

[2] Hollnagel E, editor. Resilience engineering in practice: A guidebook. Ashgate Publishing, Ltd.; 2013 Nov 1.

[3] Wears RL, Hollnagel E, Braithwaite J, editors. Resilient health care, volume 2: the resilience of everyday clinical work. Ashgate Publishing, Ltd.; 2015 Mar 28.

[4] Wears RL, Perry SJ, McFauls A. Free fall-a case study of resilience, its degradation, and recovery, in an emergency department. In2nd
International Symposium on Resilience Engineering, Juan-les-Pins, France: Mines Paris Les Presses; 2006 Nov.

[5] Anders S, Woods DD, Wears RL, et al. Limits on adaptation: modeling resilience and brittleness in hospital emergency. Learning from Diversity: Model-Based Evaluation of Opportunities for Process (Re)-Design and Increasing Company Resilience. 2006; 1.

[6] Hollnagel E. RAG-The resilience analysis grid. Resilience engineering in practice. A guidebook. Farnham, UK: Ashgate; 2011. 275-96 p. https://doi.org/10.1201/9781317065265-19

[7] Fairbanks RJ, Wears RL, Woods DD, et al. Resilience and resilience engineering in health care. Joint Commission Journal on Quality and Patient Safety. 2014 Aug 1; 40(8): 376-83. https: //doi.org/10.1016/S1553-7250(14)40049-7 
[8] Woods DD. Four concepts for resilience and the implications for the future of resilience engineering. Reliability Engineering \& System Safety. 2015 Sep 1; 141: 5-9. https ://doi.org/10.1016/j.re ss. 2015.03 .018

[9] Patriarca R, Bergström J, Di Gravio G, et al. Resilience engineering: Current status of the research and future challenges. Safety Science. 2018 Feb 1; 102: 79-100. https://doi.org/10.1016/j.ssci .2017 .10 .005

[10] Woods DD. Creating foresight: How resilience engineering can transform NASA's approach to risky decision making. Work. 2003 Oct 29; 4(2): 137-44.

[11] Khaghani F, Jazizadeh F. mD-Resilience: A Multi-Dimensional Approach for Resilience-Based Performance Assessment in Urban Transportation. Sustainability. 2020 Jan; 12(12): 4879. https : //doi.org/10.3390/su12124879

[12] Madni AM, Jackson S. Towards a conceptual framework for resilience engineering. IEEE Systems Journal. 2009 Apr 28; 3(2): 181-91. https : //doi .org/10.1109/JSYST . 2009. 2017397

[13] Menes K, Tintinalli J, Plaster L. How one Las Vegas ED saved hundreds of lives after the worst mass shooting in US history. Emergency Physicians Monthly. 2017 Nov 3.

[14] Davidson SL, Dutton RP. Lessons learned from a mass casualty incident. ASA Newsletter. 2018 Sep 1; 82(9): 8-11.

[15] Chuang S, Chang KS, Woods DD, et al. Beyond surge: Coping with mass burn casualty in the closest hospital to the Formosa Fun Coast Dust Explosion. Burns. 2019 Jun 1; 45(4): 964-73. PMid: 30598266. https ://doi.org/10.1016/j.burns.2018.12.003

[16] Woods DD. Resilience engineering: Redefining the culture of safety and risk management. Human Factors and Ergonomics Society Bulletin. 2006 Dec; 49(12): 1-3. https ://doi.org/10.1037/e721 002011-002

[17] Melmer P, Carlin M, Castater CA, et al. Mass casualty shootings and emergency preparedness: a multidisciplinary approach for an unpredictable event. Journal of Multidisciplinary Healthcare. 2019; 12: 1013. PMid: 31849477. https ://doi.org/10.2147/JMDH.S 219021

[18] VandenBerg SL, Davidson SB. Preparation for mass casualty incidents. Critical Care Nursing Clinics. 2015 Jun 1; 27(2): 157-66. PMid: 25981720. https : //doi.org/10.1016/j.cnc. 2015.02 008

[19] Biddinger PD, Baggish A, Harrington L, et al. Be prepared-the Boston Marathon and mass-casualty events. New England journal of medicine. 2013 May 23; 368(21): 1958-60. PMid: 23635020. https://doi.org/10.1056/NEJMp1305480

[20] Hollnagel E, Nemeth CP, Dekker S, editors. Resilience engineering perspectives: remaining sensitive to the possibility of failure. Ashgate Publishing, Ltd.; 2008.

[21] Nemeth C, Wears RL, Patel S, et al. Resilience is not control: healthcare, crisis management, and ICT. Cognition, Technology \& Work. 2011 Sep 1; 13(3): 189. https://doi.org/10.1007/s10111-0 11-0174-7

[22] Comfort LK. Crisis management in hindsight: Cognition, communication, coordination, and control. Public Administration Review. 2007 Dec; 67: 189-97. https://doi.org/10.1111/j.1540-6 $210.2007 .00827 . \mathrm{x}$

[23] Webb GR, Chevreau FR. Planning to improvise: the importance of creativity and flexibility in crisis response. International Journal of Emergency Management. 2006 Jan 1; 3(1): 66-72. https : //doi.org/10.1504/IJEM.2006.010282

[24] Kendra J, Wachtendorf T. Improvisation, creativity, and the art of emergency management. Understanding and responding to terrorism. 2007; 19: 324-35.
[25] Kendra J, Wachtendorf T. Creativity in emergency response to the World Trade Center disaster. Beyond September 11th: An account of post-disaster research. 2003; 121-46.

[26] Hollnagel E. The ETTO principle: efficiency-thoroughness trade-off: why things that go right sometimes go wrong. Ashgate Publishing, Ltd.; 2009.

[27] Xiao T, Sanderson P, Clayton S, et al. The ETTO principle and organisational strategies: a field study of ICU bed and staff management. Cognition, Technology \& Work. 2010 Jun 1; 12(2): 143-52. https://doi.org/10.1007/s10111-010-0147-2

[28] Duncan P, Cabral C, McCahon D, et al. Efficiency versus thoroughness in medication review: a qualitative interview study in UK primary care. British Journal of General Practice. 2019 Mar 1; 69(680): e190-8. PMid: 30745357. https://doi.org/10.3399/bjgp19 $\mathrm{X} 701321$

[29] Hollnagel E, Woods DD, Leveson N, editors. Resilience engineering: Concepts and precepts. Ashgate Publishing, Ltd.; 2006.

[30] Nathanael D, Tsagkas V, Marmaras N. Trade-offs among factors shaping operators decision-making: the case of aircraft maintenance technicians. Cognition, Technology \& Work. 2016 Nov 1; 18(4): 807-20. https ://doi .org/10.1007/s10111-016-0393-z

[31] Catchpole K. Toward the modelling of safety violations in healthcare systems. BMJ Quality \& Safety. 2013 Sep 1; 22(9): 705-9. PMid: 23580631. https://doi .org/10.1136/bmjqs-2012-001604

[32] Sujan MA, Huang H, Braithwaite J. Learning from incidents in health care: Critique from a Safety-II perspective. Safety Science. 2017 Nov 1; 99: 115-21. https://doi.org/10.1016/j.ssci.2016.08.0 05

[33] Pillay M, Borys D, Else D, et al. Safety culture and resilience engineering-Exploring theory and application in improving gold mining safety. Gravity Gold. 2010; 21-2.

[34] Sheridan TB. Risk, human error, and system resilience: fundamental ideas. Human factors. 2008 Jun; 50(3): 418-26. PMid: 18689048. https://doi .org/10.1518/001872008X250773

[35] Weick KE, Sutcliffe KM. Managing the unexpected. San Francisco: Jossey-Bass; 2001.

[36] Nemeth C, Wears R, Woods D, et al. Minding the gaps: creating resilience in health care. InAdvances in patient safety: New directions and alternative approaches (Vol. 3: Performance and tools). Agency for Healthcare Research and Quality (US). 2008 Aug.

[37] Rasoulkhani K, Mostafavi A. Resilience as an emergent property of human-infrastructure dynamics: A multi-agent simulation model for characterizing regime shifts and tipping point behaviors in infrastructure systems. PloS One. 2018 Nov 21; 13(11): e0207674. PMid: 30462719. https://doi.org/10.1371/journal.pone .0207674

[38] Epstein S. Unexampled events, resilience and PRA [probabilistic risk analysis]. InSecond Symposium on Resilience Engineering Proceedings, Juan-les-Pins, France. 2006; 8-10.

[39] Adolph L, Lafrenz B, Grauel B. Safety management systems, safety culture and resilience engineering: comparison of concepts. Proceedings of the Human Factors and Ergonomics Society Europe. 2012.

[40] Ministry of Health Singapore. Risk assessment raised to DORSCON Orange [Press release]. 2020, Feburary 7. Available from: https://www.moh.gov.sg/news-highlights/detail s/risk-assessment-raised-to-dorscon-orange

[41] Back J, Ross AJ, Duncan MD, et al. Emergency department escalation in theory and practice: a mixed-methods study using a model of organizational resilience. Annals of Emergency Medicine. 2017 Nov 1; 70(5): 659-71. PMid: 28662909. https://doi.org/10.1016/ j.annemergmed. 2017.04 .032 
[42] Sujan M, Spurgeon P, Cooke M. The role of dynamic trade-offs in creating safety-A qualitative study of handover across care boundaries in emergency care. Reliability Engineering \& System Safety. 2015 Sep 1; 141: 54-62. https://doi.org/10.1016/j.ress.2 015.03 .006

[43] Patterson M, Deutsch ES. Safety-I, safety-II and resilience engineering. Current Problems in Pediatric and Adolescent Health Care. 2015 Dec 1; 45(12): 382-9. PMid: 26549146. https://doi.org/10.1 016/j.cppeds. 2015.10.001

[44] de Carvalho PV, Righi AW, Huber GJ, et al. Reflections on work as done (WAD) and work as imagined (WAI) in an emergency response organization: A study on firefighters training exercises. Applied Ergonomics. 2018 Apr 1; 68: 28-41. PMid: 29409645. https://doi.org/10.1016/j.apergo.2017.10.016

[45] Woods DD. Essentials of resilience, revisited. InHandbook on Resilience of Socio-Technical Systems. Edward Elgar Publishing; 2019 Jan 25. https://doi .org/10.4337/9781786439376.00009

[46] Bergström J, Van Winsen R, Henriqson E. On the rationale of resilience in the domain of safety: A literature review. Reliability Engineering \& System Safety. 2015 Sep 1; 141: 131-41. https: //doi.org/10.1016/j.ress.2015.03.008
[47] Gopalakrishna G, Choo P, Leo YS, et al. SARS transmission and hospital containment. Emerging Infectious Diseases. 2004 Mar; 10(3): 395. PMid: 15109403. https://doi.org/10.3201/eid1003.03 0650

[48] Provan DJ, Woods DD, Dekker SW, et al. Safety II professionals: how resilience engineering can transform safety practice. Reliability Engineering \& System Safety. 2020 Mar 1; 195: 106740. https://doi.org/10.1016/j.ress.2019.106740

[49] Pillay M, Morel G. Measuring Resilience Engineering: An Integrative Review and Framework for Bench-Marking Organisational Safety. Safety. 2020 Sep; 6(3): 37. https://doi.org/10.3390/ safety6030037

[50] Tan BY, Chew NW, Lee GK, et al. Psychological impact of the COVID-19 pandemic on health care workers in Singapore. Annals of Internal Medicine. 2020 Apr 6. PMid: 32251513. https: //doi.org/10.7326/M20-1083

[51] Hollnagel E, Fujita Y. The Fukushima disaster-systemic failures as the lack of resilience. Nuclear Engineering and Technology. $2013 \mathrm{Feb}$ 1; 45(1): 13-20. https://doi.org/10.5516/NET .03.2011.078

[52] Quarantelli EL. Disaster crisis management: A summary of research findings. Journal of Management Studies. 1988 Jul; 25(4): 373-85. https://doi.org/10.1111/j.1467-6486.1988.tb00043.x 